\title{
Sarcoma Care Collaborations: Models for patient centered sub- specialty oncology care in the COVID-19 era and beyond
}

\author{
Michael J. Wagner, MD ${ }^{1}$, Majid Chalian, $\mathrm{MD}^{2}$, Robert Ricciotti, $\mathrm{MD}^{3}$, Jesse L. Roberts, MD ${ }^{4}$, \\ Matthew J. Thompson, $\mathrm{MD}^{4}$, Stephanie Schaub, $\mathrm{MD}^{5}$ \\ ${ }^{1}$ Medical Oncology, University of Washington; Clinical Research Division, Fred Hutchinson Cancer Center, 2 Radiology, University of Washington, \\ 3 Pathology, University of Washington, ${ }^{4}$ Orthopaedics and Sports Medicine, University of Washington, 5 Radiation Oncology, University of Washington \\ Keywords: Sarcoma, Telehealth, academic-community partnership, COVID-19 \\ https://doi.org/10.53876/001c.32477
}

International Journal of Cancer Care and Delivery

Vol. 2, Issue 1, 2022

Sarcomas are a group of rare cancers that often require sub-specialty multidisciplinary opinion for appropriate management. Partnerships between referral sarcoma centers and community providers are critical for ensuring access to evidence-based care across multiple care settings. Herein, we discuss sarcoma specific considerations for academic-community partnerships across the cancer care spectrum and how these relationships have been impacted by the COVID-19 pandemic.

\section{BACKGROUND}

There are over 17,000 people diagnosed in the US every year with cancers of soft tissue and bone, and over 7,000 deaths are attributed to these cancers. ${ }^{1}$ Improvements in surgical and radiation techniques and multiple new histology specific drug approvals over the past few years have led to a rapid pace of change in sarcoma management. The management of sarcoma for both localized and metastatic disease is becoming more nuanced requiring a personalized approach for each individual patient. NCCN guidelines state "Prior to the initiation of therapy, it is highly recommended that all patients be evaluated and managed by a multidisciplinary team with expertise and experience in sarcoma." ${ }^{2}$ To accomplish this, the input of specialists across the cancer care spectrum including pathology, surgery, radiation oncology, medical oncology, and radiology is often required. This creates logistical challenges both for patients and providers. For many patients, the collaboration between academic and community providers is critical for the timely delivery of sarcoma care. Depending on patient preference, available resources, and the recommended treatments, we adopt one of several paradigms for collaboration with community providers. Herein, we discuss the importance of these collaborative relationships for multidisciplinary sarcoma care and how we have approached this in the COVID-19 era.

Academic- community partnerships are perhaps most relevant for patients in rural areas without easy access to a sarcoma specialty center. Patients in rural areas have longer travel times to sub-specialty cancer centers. ${ }^{3}$ It is a potentially huge burden to ask a patient to travel long distances at great expense, let alone while they are undergoing cancer treatment. The distance needed to travel to a treating oncologist is associated with a lower likelihood of receiving chemotherapy in patients with resected colon cancer. ${ }^{4}$ In Ewing sarcoma, delays in transitions from one modality to another (e.g. from diagnosis to chemotherapy initiation, to surgery, and back to adjuvant chemotherapy) are associated with inferior outcomes. ${ }^{5}$ Prior to the widespread adoption of telemedicine, patients would generally have to travel to get a sarcoma specialty opinion. Telemedicine and enhanced collaboration between community providers and specialty centers holds the potential to reduce unnecessary travel, expense, anxiety, and delayed care by allowing for remote review of imaging and early guidance of appropriate workup. Although collaborative care relationships extend beyond patients seen in formal consultation, a full evaluation of pathology and imaging is frequently required to offer a full opinion.

The COVID-19 pandemic opened avenues for streamlined collaboration between sarcoma specialists and community providers. Prior to the pandemic, telemedicine was rarely used in oncology care delivery. When COVID-19 first emerged, patients with sarcoma had high rates of COVID-19 complications. ${ }^{6}$ Many patients faced a dilemma of whether to travel for care or to seek care closer to home. Since patients with sarcoma may live far from a high-volume sarcoma center, telemedicine promises to ease the burden of getting an expert opinion and allow for expedited sub-specialty input. Indeed, telemedicine became more common after more permissive policies were put in place. ${ }^{7}$ Challenges introduced by telemedicine such as the technical aspects of connecting can be overcome with clear instructions and technical support. This is aided by embedding telemedicine platforms into the electronic medical record which streamlines the delivery of care and also tackles the question of concerns about privacy by ensuring that the virtual connection is HIPAA compliant.

Like with other aspects of care, the COVID-19 pandemic triggered a move towards virtual multidisciplinary tumor boards. ${ }^{8}$ Discussion at a sarcoma sub-specialty multidis- 
ciplinary tumor board is associated with increased adherence to clinical practice guidelines and improved survival for sarcoma patients. ${ }^{9}$ Suggested guidelines for a structured tumor board for sarcoma have been previously described, with a delineation of benefits that includes improved quality of care, more efficient use of scarce resources, and a documented plan of care to allow for improved collaborative care and communication among providers both within and from outside the specialty institution. ${ }^{10}$ Even though this opens up the opportunity for outside providers to join the tumor board, in virtually all cases time constraints and other obligations mean that outside providers are not able to join in real-time. As was done prior to the pandemic, results of the tumor board discussion are conveyed to referring physicians through other means.

Below, we discuss each of the key physician specialties involved in our tumor board and sarcoma specific considerations for academic-community partnerships:

\section{PATHOLOGY}

In part due to the rarity of any individual sarcoma diagnosis, pathology review by a sarcoma specialist pathologist is critical prior to initiation of any treatment plan. There is a disturbingly high level of discordance between non-expert pathologist review and centralized specialist review. Only $56 \%$ of cases in one large study had complete agreement between both pathologists. There was partial agreement in another 38\% with the most common discrepancy concerning tumor grading. Major discrepancies, such as benign vs. malignant diagnoses or a different subtype requiring different management, were observed in $8 \%$ of cases. ${ }^{11}$ These differences may mean the difference between no chemotherapy, for example for a conventional chondrosarcoma, or intensive combination chemotherapy such as what is required for osteosarcoma. ${ }^{12}$

\section{CHEMOTHERAPY}

In general terms, there are three paradigms by which we work with local providers for the management of sarcoma chemotherapy. Some patients receive all care with their local oncologist who will reach out as needed for sarcoma specialty input. Some sarcoma diagnoses require long courses of complicated multi-agent chemotherapy. This is especially true for some highly curable sarcomas that typically present in adolescents and young adults such as Ewing sarcoma, rhabdomyosarcoma, and osteosarcoma. Community sites are not always set up with the resources to deliver these regimens or to manage potential toxicities that may arise. Continued education between specialist and community provider is an opportunity for collaboration and may ease the burden on patients if local centers are able to administer complicated chemotherapy regimens typically given at higher volume sarcoma centers. Higher sarcoma volume and familiarity with these subtype specific regimens may lead some to prefer treatment at a sarcoma center. Other patients opt for a combination of care to get certain standard of care treatments locally, with regular visits at our center usually coinciding with decision time- points like scan reviews. With the challenges associated with travel compounded by the COVID-19 pandemic, this option has become more popular. It is less burdensome to patients who can often be seen via telemedicine without ever incurring the time and financial cost of traveling for in person evaluation. Most patients receiving standard of care chemotherapy for metastatic soft tissue sarcoma can receive it at any cancer center but still benefit from specialty consultation now that subsequent line therapies are becoming more and more subtype specific. Finally, some patients travel for all their care either by choice or due to a lack of local resources.

Clinical trials for sarcoma are more commonly available at referral sites. Since clinical trial availability can come and go with little notice, communication between providers is critical to ensure that patients are aware of the trial options and can access trials as best as possible, while minimizing the travel burden when no trials are available. Due to the need for close principal investigator oversight of clinical trials, clinical trial participation by necessity requires frequent travel to referral centers. However, certain testing such as routine toxicity labs and imaging for response evaluations may be done locally in an effort to minimize the travel burden when feasible. A large survey of oncologists suggested that about half of all clinic visits could potentially be virtual. ${ }^{13}$ Similar to local labs and testing, telemedicine has the potential to revolutionize access to specialty care and clinical trials by allowing for virtual toxicity checks.

\section{RADIATION}

Radiation therapy for sarcomas often consists of a protracted course of $5-6.5$ weeks of daily treatment which can result in significant logistical, financial, and social support hardships for patients. Indications, treatment volumes, recommended dose, and treatment modality are becoming more nuanced based on the grade, histology, location, and pattern of spread. Consensus and target delineation guidelines for soft tissue sarcomas and retroperitoneal sarcomas provide a framework to promote standardization of quality of care delivered between referral and community centers. ${ }^{14}$ In addition, many cooperative group protocols in the pediatric and adult setting for sarcoma require centralized quality assurance peer review prior to treatment, and adherence to protocol guidelines has been shown to result in improved outcomes. ${ }^{15}$

In practice, with the increased access to referral centers via telehealth or through virtual sarcoma tumor boards, we can more provide timely radiotherapy recommendations, including often personal discussion with the treating community-based radiation oncologist, particularly when a photon or electron based treatment course is indicated which are more widely available technologies. Certain cases may benefit from treatment at more specialized referral centers using advanced treatment modalities such as proton therapy, particularly for pediatric and young adults, head and neck, spine and base of skull tumors, and in re-irradiation cases to minimize long-term morbidity. ${ }^{16}$ Collaboration with the community based radiation oncologist for 


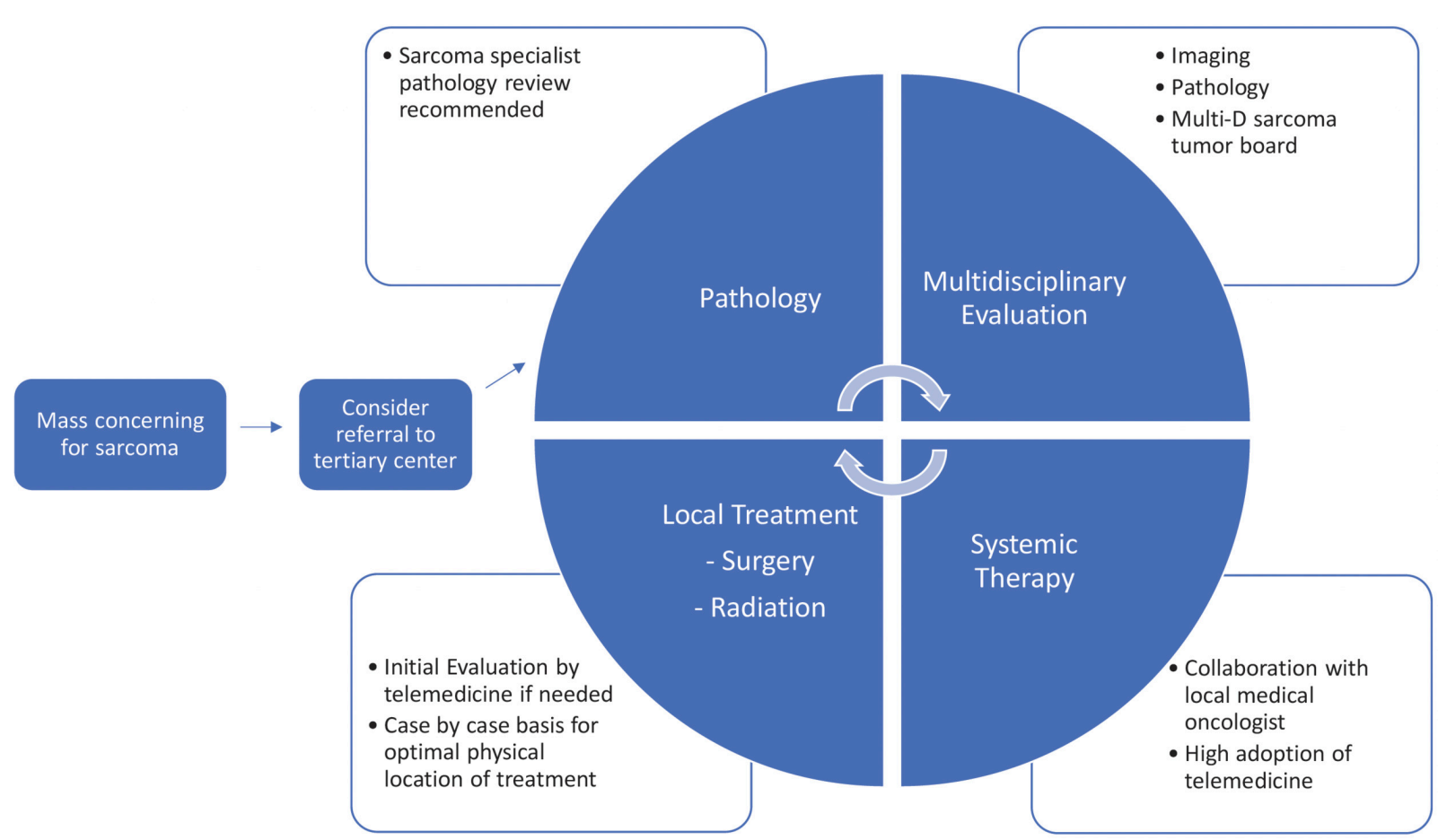

Figure 1. Schematic demonstrating general flow for management of a patient with sarcoma.

comparison plans between protons and photons have been utilized as a means to evaluate the potential medical necessity for proton therapy.

\section{SURGERY}

Surgical outcomes are dependent on surgeon volume. ${ }^{17}$ For sarcoma, adherence to clinical practice guidelines for surgery correlates with improved progression free survival. ${ }^{18}$ When a soft tissue or bone mass is initially thought to be benign, sometimes the initial attempt to resect a mass can be a non-oncologic surgery that then requires additional procedures when final pathology reveals a more aggressive sarcoma. These so called initial “whoops” surgeries are associated with a need for more extensive surgeries down the road ${ }^{19}$. Even biopsies performed at a referring institution rather than a dedicated treatment center have been associated with a 2-12 times greater chance of errors, complications, or changes in the course and/or outcome of treatment. ${ }^{20} \mathrm{~A}$ misplaced biopsy or "whoops" procedure can compromise the possibility of limb salvage local control surgery. We, therefore, recommend that local providers reach out early in the care of a patient with sarcoma, or even undiagnosed soft tissue or bone masses, to guide appropriate workup and to assess for a potential benefit of sub-specialty sarcoma evaluation. Many patients with sarcoma have one chance for cure. A common and easy-to-remember guide is that a growing soft tissue mass the size of a golf ball likely warrants evaluation by a sarcoma specialist. $^{21}$

\section{RADIOLOGY}

Sarcomas could occur at diverse anatomic locations throughout the body. The heterogeneity of subtypes and locations makes anatomic evaluation and assessment of adjacent structures challenging. In addition, despite the advancement of localized therapies such as radiotherapy (RT) and surgery, sarcoma can recur in approximately $50 \%$ of cases. ${ }^{22}$ Imaging with the most appropriate protocols for the patient's situation is the first step for effective imaging. Clear indications for imaging from the ordering provider, and communication from the requisitioning radiologist to resolve any remaining uncertainty, are critical to maximizing downstream interpretation of the imaging. There is a high level of discordance between community radiologists and sub-specialty radiologists at referral sarcoma centers on the interpretation of sarcoma imaging. The subspecialty second-opinion consultation was more accurate than outside reports in $82.0 \%$ of examinations when pathologic confirmation was made. A moderate rate (26.2\%) of discrepant interpretations was noted between outside and subspecialty second-opinion consultation provided on MSK imaging examinations on tumor cases $(36.3 \%){ }^{23}$ These challenges highlight the importance of inter-disciplinary collaboration for optimal patient centered sarcoma care. This collaboration has been revolutionized during the COVID-19 pandemic with remote meetings and more streamlined conferences and discussions.

\section{ADOLESCENT AND YOUNG ADULT ONCOLOGY (AYA)}

AYA patients face a unique set of challenges when confronting cancer care. They are often students, or just starting a career when given a cancer diagnosis. Many are con- 
sidering starting families, with fertility preservation as a top goal prior to initiating gonadotoxic therapy. While supportive care services are critical for all sarcoma patients, this is especially true for the AYA population who may be less able to travel. In addition to formal support services, social media and patient advocacy networks can help patients with rare diagnoses learn about and cope with their disease and treatment. Telemedicine and establishing a sarcoma center while receiving most care locally may improve access to supportive services for patients who are otherwise unable to move away from their school, job, or families.

\section{CONCLUSION}

The practice changes resulting from the COVID-19 era such as increased adoption of telemedicine have shed light on how we might optimize communication and collaboration between community sites and referral centers. These collaborative care relationships expedite patient care and allow for adherence to clinical practice guidelines. The portability of pathology and imaging results without a requirement for patient travel has the promise of revolutionizing care structures for rare cancers. Treatment at a referral center has been shown to normalize oncologic outcomes regardless of socioeconomic status, ${ }^{24}$ and the goal would be that by maintaining the flow of communication and taking advantage of emerging technologies, all sarcoma patients regardless of location will have access to multidisciplinary sarcoma care.

Submitted: December 06, 2021 PST, Accepted: February 02, 2022 PST 


\section{REFERENCES}

1. Siegel RL, Miller KD, Fuchs HE, Jemal A. Cancer Statistics, 2021. CA Cancer J Clin. 2021;71(1):7-33. do i:10.3322/caac.21654

2. von Mehren M, Kane JM, Bui MM, et al. NCCN Guidelines Insights: Soft Tissue Sarcoma, Version 1.2021. J Natl Compr Canc Netw. 2020;18(12):1604-1612. doi:10.6004/jnccn.2020.0058

3. Onega T, Duell EJ, Shi X, Wang D, Demidenko E, Goodman D. Geographic access to cancer care in the U.S. Cancer. 2008;112(4):909-918. doi:10.1002/cncr.2 $\underline{3229}$

4. Lin CC, Bruinooge SS, Kirkwood MK, et al. Association Between Geographic Access to Cancer Care, Insurance, and Receipt of Chemotherapy: Geographic Distribution of Oncologists and Travel Distance. Journal of Clinical Oncology. 2015;33(28):3177-3185. doi:10.1200/JCO.2015.61.155 $\underline{8}$

5. Dirksen U, Ranft A, Baumhoer D, et al. Association of treatment delays with an unfavorable outcome in patients with localized Ewing sarcoma: A retrospective analysis of data from the GPOH EuroE.W.I.N.G.99 trial. Journal of Clinical Oncology. 2021;39(15_suppl):11502-11502. doi:10.1200/JCO.202 1.39 .15 suppl.11502

6. Wagner MJ, Pollack SM, Cranmer LD, et al. Outcomes of Patients with Sarcoma and COVID-19 Infection: A Single Institution Cohort Analysis. Cancer Invest. 2021;39(4):315-320. doi:10.1080/07357 907.2021.1903914

7. Zon RT, Kennedy EB, Adelson K, et al. Telehealth in Oncology: ASCO Standards and Practice

Recommendations. JCO Oncology Practice. 2021;17(9):546-564. doi:10.1200/OP.21.00438

8. Dharmarajan H, Anderson JL, Kim S, et al. Transition to a virtual multidisciplinary tumor board during the COVID-19 pandemic: University of Pittsburgh experience. Head Neck. 2020;42(6):1310-1316. doi:10.1002/hed.26195

9. Blay JY, Soibinet P, Penel N, et al. Improved survival using specialized multidisciplinary board in sarcoma patients. Ann Oncol 2017.

2017;28(11):2852-2859. doi:10.1093/annonc/mdx484

10. Siegel GW, Biermann JS, Chugh R, et al. The multidisciplinary management of bone and soft tissue sarcoma: an essential organizational framework. J Multidiscip Healthc. 2015;8:109-115. do i:10.2147/JMDH.S49805
11. Ray-Coquard I, Montesco MC, Coindre JM, et al. Sarcoma: concordance between initial diagnosis and centralized expert review in a population-based study within three European regions. Annals of Oncology. 2012;23(9):2442-2449. doi:10.1093/annonc/mdr610

12. Wagner MJ, Livingston JA, Patel SR, Benjamin RS. Chemotherapy for Bone Sarcoma in Adults. J Oncol Pract. 2016;12(3):208-216. doi:10.1200/JOP.2015.0099 $\underline{44}$

13. Tevaarwerk AJ, Chandereng T, Osterman T, et al. Oncologist Perspectives on Telemedicine for Patients With Cancer: A National Comprehensive Cancer Network Survey. JCO Oncology Practice. 2021;17(9):e1318-e1326. doi:10.1200/OP.21.00195

14. Salerno KE, Alektiar KM, Baldini EH, et al. Radiation Therapy for Treatment of Soft Tissue Sarcoma in Adults: Executive Summary of an ASTRO Clinical Practice Guideline. Pract Radiat Oncol. 2021;11(5):339-351. doi:10.1016/j.prro.2021.04.005

15. Haas RLM, Stelmes JJ, Zaffaroni F, et al. Critical impact of radiotherapy protocol compliance and quality in the treatment of retroperitoneal sarcomas: Results from the 62092-22092 STRASS trial. Journal of Clinical Oncology. 2021;39(15 suppl):11566-11566. doi:10.1200/JCO.2021.39.15 suppl.11566

16. DeLaney TF, Haas RL. Innovative radiotherapy of sarcoma: Proton beam radiation. Eur J Cancer. 2016;62:112-123. doi:10.1016/i.ejca.2016.04.015

17. Birkmeyer JD, Stukel TA, Siewers AE, Goodney PP, Wennberg DE, Lucas FL. Surgeon Volume and Operative Mortality in the United States. New England Journal of Medicine. 2003;349(22):2117-2127. doi:10.1056/NEJMsa035205

18. Derbel O, Heudel PE, Cropet C, et al. Survival impact of centralization and clinical guidelines for soft tissue sarcoma (A prospective and exhaustive population-based cohort). PloS one. 2017;12(2):e0158406-e0158406. doi:10.1371/journa l.pone.0158406

19. Traub F, Griffin AM, Wunder JS, Ferguson PC. Influence of unplanned excisions on the outcomes of patients with stage III extremity soft-tissue sarcoma. Cancer. 2018;124(19):3868-3875. doi:10.1002/cncr.31 $\underline{648}$ 
20. Mankin HJ, Mankin CJ, Simon MA. The hazards of the biopsy, revisited. Members of the Musculoskeletal Tumor Society. J Bone Joint Surg Am.

1996;78(5):656-663. doi:10.2106/00004623-19960500 $\underline{0-00004}$

21. Nandra R, Forsberg J, Grimer R. If your lump is bigger than a golf ball and growing, think Sarcoma. Eur J Surg Oncol. 2015;41(10):1400-1405. doi:10.1016/ j.ejso.2015.05.017

22. Rothermundt C, Whelan JS, Dileo P, et al. What is the role of routine follow-up for localised limb soft tissue sarcomas? A retrospective analysis of 174 patients. Br J Cancer. 2014;110(10):2420-2426. doi:1 0.1038/bjc.2014.200
23. Chalian M, Del Grande F, Thakkar RS, Jalali SF, Chhabra A, Carrino JA. Second-Opinion Subspecialty Consultations in Musculoskeletal Radiology. AJR Am J Roentgenol. 2016;206(6):1217-1221. doi:10.2214/ajr.1 $\underline{5.14540}$

24. Eastman BM, Hippe DS, Wootton LS, et al. Socioeconomic factors do not affect overall survival in soft tissue sarcoma when patients treated at a single high-volume center. BMC Cancer. 2021;21(1):620. do $\mathrm{i}: 10.1186 / \mathrm{s} 12885-021-08352-\mathrm{z}$ 